ఠ

\title{
Anti-inflammatory effects of silver-polyvinyl pyrrolidone (Ag-PVP) nanoparticles in mouse macrophages infected with live Chlamydia trachomatis
}

\author{
This article was published in the following Dove Press journal: \\ International Journal of Nanomedicine \\ 6 July 2013 \\ Number of times this article has been viewed
}

\section{Abebayehu N Yilma \\ Shree R Singh \\ Saurabh Dixit \\ Vida A Dennis}

Center for Nanobiotechnology and Life Sciences Research, Alabama State University, Montgomery, AL, USA
Correspondence: Vida A Dennis Department of Biological Sciences, Alabama State University, 1627 Hall Street, Montgomery, AL, 36I04, USA Tel +l 3342298447

Email vdennis@alasu.edu

\begin{abstract}
Chlamydia trachomatis is a very common sexually transmissible infection in both developing and developed countries. A hallmark of C. trachomatis infection is the induction of severe inflammatory responses which play critical roles in its pathogenesis. Antibiotics are the only treatment option currently available for controlling C. trachomatis infection; however, they are efficacious only when administered early after an infection. The objectives of this study are to explore alternative strategies in the control and regulation of inflammatory responses triggered by a C. trachomatis infection. We employed silver-polyvinyl pyrrolidone (Ag-PVP) nanoparticles, which have been shown to possess anti-inflammatory properties, as our target and the in vitro mouse $\mathrm{J} 774$ macrophage model of $C$. trachomatis infection. Our hypothesis is that small sizes of Ag-PVP nanoparticles will control inflammatory mediators triggered by a $C$. trachomatis infection. Cytotoxicity studies using Ag-PVP nanoparticles of 10, 20, and $80 \mathrm{~nm}$ sizes revealed $>80 \%$ macrophage viability up to a concentration of $6.25 \mu \mathrm{g} / \mathrm{mL}$, with the $10 \mathrm{~nm}$ size being the least toxic. All sizes of Ag-PVP nanoparticles, especially the $10 \mathrm{~nm}$ size, reduced the levels of the prototypic cytokines, tumor necrosis factor (TNF) and interleukin (IL)-6, as elicited from C. trachomatis infected macrophages. Additionally, Ag-PVP nanoparticles $(10 \mathrm{~nm})$ selectively inhibited a broad spectrum of other cytokines and chemokines produced by infected macrophages. Of significance, Ag-PVP nanoparticles $(10 \mathrm{~nm})$ caused perturbations in a variety of upstream (toll like receptor 2 [TLR2], nucleotide-binding oligomerization-protein 2 [NOD2], cluster of differentiation [CD]40, CD80, and CD86) and downstream (IL-1 receptor-associated kinase 3 [IRAK3] and matrix metallopeptidase 9 [MMP9]) inflammatory signaling pathways by downregulating their messenger ribonucleic acid (mRNA) gene transcript expressions as induced by C. trachomatis in macrophages. Collectively, our data provides further evidence for the anti-inflammatory properties of Ag-PVP nanoparticles, and opens new possibilities for smaller sizes of Ag-PVP nanoparticles to be employed as regulators of inflammatory responses induced by $C$. trachomatis.
\end{abstract}

Keywords: silver nanoparticles, cytokines, chemokines, TLR2, NOD2, bacteria, sexually transmitted disease

\section{Introduction}

Chlamydia trachomatis is an obligate intracellular bacterium which is implicated in a wide spectrum of diseases in humans and other mammals worldwide. ${ }^{1-3}$ In humans, it is the leading cause of sexually transmitted bacterial diseases in developed countries and is the main cause of blindness in developing countries. ${ }^{3}$ The pathogen exists in two distinct morphological forms: the replicative, intracellular reticulate body $(\mathrm{RB})$ and the infectious but metabolically inactive elementary body (EB). Infection is initiated 
by adherence of EBs to the host cell receptors through its ligands, including the heat shock protein 70 (Hsp70) and outer membrane protein 2 (omp2), after which they are internalized and form inclusion compartments. ${ }^{4-8}$ Within the inclusions, EBs differentiate into RBs for proliferation and between 48 and 72 hours post-infection, the RBs redifferentiate into EBs and are released into the extracellular space to start a new round of infection.

Mammalian cells sense the presence of invading microbial pathogens through recognition of pathogen associated molecular patterns (PAMPs). Toll like receptors (TLRs) are the major membrane bound receptors that bind PAMPs and to date, at least 11 have been identified in mammals. In addition to TLRs, mammalian cells have cytosolic surveillance for PAMPs via the nucleotide binding site/leucine-rich repeat (NBS/LRR) proteins, nucleotide binding oligomerization domain protein (NOD) 1 and 2 . NOD1 is activated by a peptidoglycan motif found in gram negative bacteria, while NOD2 binds muramyl dipeptides. ${ }^{9-12}$ After recognition of microbial product, either by TLRs or NODs, various signaling events are triggered to allow nuclear translocation of nuclear factor kappa B (NF-кB) and subsequent upregulation of inflammatory mediators. Therefore, effective recognition of the pathogen is critical for host defense responses and initiation of the inflammatory response.

Inflammation is the primary response to infection that functions to clear the infectious agent and promote tissue repair. It is characterized by the sequential release of a series of mediators including cytokines, chemokines, and growth factors that regulate vascular permeability and recruitment of blood borne leukocytes. Increased vascular permeability also results in excessive plasma proteins that further amplify the inflammatory reaction. Therefore, the process of inflammation is intimately linked to host immune responses in an effort to resolve the infection. However, such efforts are not always successful in eliminating the infection as some pathogens persist and elicit chronic immune responses by misdirecting the immune responses to result in immunopathological diseases. Indeed, diseases caused by Chlamydia are thought to be mediated by the ensuing immunopathology. ${ }^{13}$

There is much evidence to support that blindness and sterility caused by $C$. trachomatis are the result of inflammation based pathology. ${ }^{13,14}$ At the sites of $C$. trachomatis infection, intense inflammation is characterized by redness, edema, and mucopurulent discharge. ${ }^{14}$ An intense and chronic inflammatory response and follicle necrosis arises when there is damage to the epithelium, and this is followed by local epithelial cell proliferation and scar formation. ${ }^{14}$ As the infection prolongs, plasma cells and macrophages are continuously recruited and at the same time allow the release of more immune cells both from adaptive and innate immune responses. ${ }^{15,16}$ Many years after the infection, the physical consequences of the disease emerge which ultimately lead to visual impairment and infertility.

Antibiotics are the only available intervention strategy to treat Chlamydia infection; however, these antibiotics are effective if they are taken early during the infection. However, the silent nature of the disease does not permit patients to seek early antibiotic treatment. When Chlamydia persists, antibiotics fail to destroy the pathogen, thereby resulting in continuous damage to the host. ${ }^{17}$ In a community wide antibiotic treatment trial for trachoma, both reinfection and persistent infection in humans were documented indicating loss of antibiotic efficacy. ${ }^{18}$ Reactivation of persistent Chlamydia is also a problem that prevents inhibition of Chlamydia replication and weakens the effectiveness of antibiotics..$^{20,21}$ Therefore, it is necessary to explore alternative treatment strategies to reduce the excessive production of inflammatory mediators.

The advancement of nanotechnology in medicinal applications allows opportunities to explore metal nanoparticles in the field of biology. Silver has long been known for its antimicrobial properties. ${ }^{23-27}$ Silver nanoparticles have been widely explored for their antimicrobial potential against both gram-negative and gram-positive bacteria, ${ }^{26,27}$ and as antiviral agents against human immunodeficiency virus $-1,{ }^{28}$ hepatitis $B$ virus, ${ }^{29}$ respiratory syncytial virus, ${ }^{30}$ herpes simplex virus type-1, ${ }^{31}$ and monkeypox virus plaque formation. ${ }^{32}$ Furthermore, silver nanoparticles were shown to reduce oxidative stress, and were recently described as an environmentally friendly approach to synthesize oil-based paints with antimicrobial properties. ${ }^{33,34}$

Silver nanoparticles also possess anti-inflammatory properties. Previously, it has been shown that silver nanoparticles decreased wound inflammation in a thermal injury animal model. ${ }^{35,36}$ Silver nanoparticles have also been shown to decrease the levels of inflammatory markers in animals in vivo. ${ }^{37}$ Collectively, the results from these studies suggest that silver nanoparticles may be involved in altering or suppressing inflammatory events during microbial interventions, and thus play a critical role in balancing the level of inflammatory mediators secreted during infection. The ultimate goal of this study is to further enhance our knowledge of silver nanoparticles as an anti-inflammatory agent, and to provide insights into whether silver nanoparticles can be used to prevent excessive inflammatory responses, as triggered by live C. trachomatis 
in mouse macrophages. Herein, we used three different sizes of silver-polyvinyl pyrrolidone (Ag-PVP) nanoparticles to investigate (1) the toxic effect of Ag-PVP nanoparticles to mouse J774 macrophages, (2) the ability of Ag-PVP nanoparticles to diminish the production levels of cytokines and chemokines as elicited by $C$. trachomatis infected macrophages, and (3) the effect of Ag-PVP nanoparticles on the messenger RNA (mRNA) gene transcript expression of macrophage receptors and other downstream inflammatory genes. The data from this study provides evidence for a role of Ag-PVP nanoparticles in regulating inflammatory mediators in C. trachomatis infected macrophages, and promotes the novel idea of Ag-PVP nanoparticles as a potential antiinflammatory molecule against $C$. trachomatis in vivo.

\section{Materials and methods Cell culture and infectivity}

Mouse J774 macrophages were obtained from the American Type Culture Collection ([ATCC], Manassas, VA, USA) and cultured as already described. ${ }^{19}$ C. trachomatis MoPn Nigg II was purchased from ATCC (ATCC \#VR-123) and propagated as reported. ${ }^{19}$ To establish infection, macrophages $\left(10^{6}\right.$ cells/ well) were seeded in 24 well plates for 24 hours after which they were infected with live $C$. trachomatis infectious particles $\left(10^{5}\right)$ in $500 \mu \mathrm{L}$ of growth media/well and incubated at $37^{\circ} \mathrm{C}$ with $5 \% \mathrm{CO}_{2}$ for 48 hours. The optimum bacteria dose and duration of infection were determined as reported. ${ }^{19}$

\section{Preparation of Ag-PVP nanoparticles}

Ag-PVP nanoparticles at 20 and $80 \mathrm{~nm}$ were purchased from Nanostructure and Amorphous Materials, Inc (Houston, TX, USA). Ag-PVP nanoparticles of $10 \mathrm{~nm}$ was previously synthesized in our laboratory. ${ }^{30}$ We used Ag-PVP nanoparticles because they have been shown to be more stable and uniform in size than other coated nanoparticles, and this contributes greatly to their effectiveness in biomedical applications. ${ }^{30}$

\section{Cytotoxicity studies}

The cytotoxicity of Ag-PVP nanoparticles to mouse J774 macrophages was measured using the 3-(4,5-dimeth ylthiazol-2-yl)-2,5-diphenyl tetrazolium bromide (MTT) dye reduction assay and the Cell-Titer 96 Cell Proliferation Assay kit (Promega, Madison, WI, USA). Mouse J774 macrophages were seeded in a 96 well plate at a density of $10^{5}$ cells/well in $50 \mu \mathrm{L}$ of media and incubated overnight at $37^{\circ} \mathrm{C}$ with $5 \% \mathrm{CO}_{2}$. Ag-PVP nanoparticles of 10, 20, and $80 \mathrm{~nm}$ were added to cells in concentrations ranging from 0.39 to $200 \mu \mathrm{g} / \mathrm{mL}$ and after 48 hours, supernatants were removed, and cells washed two times with sterile phosphate buffered saline (PBS). MTT dye solution $(15 \mu \mathrm{L})$ was added to each well, and cells were further incubated for 3 hours at $37^{\circ} \mathrm{C}$ with $5 \% \mathrm{CO}_{2}$. To stop the reaction, $100 \mu \mathrm{L}$ of solubilization solution/stop mixture was added to each well and plates were incubated for 30 minutes at room temperature. Absorbance at $570 \mathrm{~nm}$ was measured using a TECAN Sunrise plate reader (TECAN US Inc, Durham, NC, USA). Percent cell viability was obtained using the optical density readings of Ag-PVP nanoparticle treated cells compared to those of normal cells (control), where percent viability $=[\mathrm{A}]_{\text {test }} /[\mathrm{A}]_{\text {control }} \times 100$, and where $[\mathrm{A}]_{\text {test }}$ is the absorbance of the test sample and $[\mathrm{A}]_{\text {control }}$ is the absorbance of the control sample.

\section{Cytokine and chemokine measurement}

Mouse $\mathrm{J} 774$ macrophages were infected with $C$. trachomatis for 2 days as indicated above after which the media were replaced with fresh media containing Ag-PVP nanoparticles of either 10, 20 and $80 \mathrm{~nm}$ sizes at concentrations of 0.39 , $0.79,1.5,3.12$, and $6.25 \mu \mathrm{g} / \mathrm{mL}$. For some experiments, AgPVP nanoparticles (10 nm) was used at $2.5 \mu \mathrm{g} / \mathrm{mL}$. Cell-free supernatants were collected after an additional 48 hour incubation following centrifugation at $450 \mathrm{~g}$ for 10 minutes at $4^{\circ} \mathrm{C}$, and stored at $-80^{\circ} \mathrm{C}$ until used. Cytokines and chemokines were quantified using single (BD-Biosciences, San Jose, CA, USA) and multiplex (EMD Millipore Corporation, Billerica, MA, USA) ELISAs. ${ }^{19,38}$

\section{Confocal microscopy}

To provide physical evidence for the secretion of interleukin (IL)-6 and tumor necrosis factor (TNF), mouse J774 macrophages $\left(2.5 \times 10^{4}\right.$ cells/well $)$ were left uninfected or infected with C. trachomatis $\left(2.5 \times 10^{3}\right.$ inclusion forming units [IFU]/ well) using 6 well chamber slides. After 2 days infection, the media were replaced with fresh media supplemented with or without Ag-PVP nanoparticles and allowed to incubate for an additional 48 hours. Post Ag-PVP nanoparticle incubation, supernatants were removed, cells were fixed with $2 \%$ paraformaldehyde (PFA) (USB Corporation, Cleveland, OH, USA) and then permeabilized with $100 \%$ methanol. The cells were immunostained with anti-mouse TNF FITC (fluorescein isothiocyanate) and IL-6 PE (phycoerythrin) antibodies (BD Biosciences, San Jose, CA, USA) overnight at $4^{\circ} \mathrm{C}$, washed three times with $1 \times$ PBS, and stained with DAPI (4',6-diamidino2-phenylindole) combined with an antifade mounting solution (Life Technology, Carlsbad, CA, USA). Slides were examined with a Nikon Eclipse Ti Confocal Microscope (Nikon Instrument, Melville, NY, USA) at 40× magnification. 


\section{Flow cytometry}

Mouse J774 macrophages $\left(10^{6}\right.$ cells $\left./ \mathrm{mL}\right)$ were left uninfected or infected with $C$. trachomatis and after a 48 hour infection period, the media were removed and replenished with fresh media containing Ag-PVP nanoparticles and further incubated for 48 hours. The cells were blocked with Fc blocking antibody (BD Bioscience) in FACS (Fluorescence-activated cell sorting) buffer (PBS/0.1\% $\mathrm{NaN}_{3} / 1.0 \%$ fetal bovine serum) for 15 minutes at $4{ }^{\circ} \mathrm{C}$, washed 2 times, and stained with fluorochrome-conjugated antibodies (50 $\mu \mathrm{L}$ in FACS buffer) against mouse CD80 (cluster of differentiation 80) (PE-Cy7) and CD86 allophycocyanin (APC) (eBiosciences, San Diego, CA, USA). The optimum concentration for all fluorochromes was predetermined in our laboratory. Cells were incubated with fluorochrome antibodies for 30 minutes at $4^{\circ} \mathrm{C}$, washed 2 times, and then fixed using 2\% PFA solution. Data were acquired on a BD FACS Canto II flow cytometer (BD Bioscience) with at least $10^{5}$ events for each sample and analyzed using Flow-Jo software (Tree Star, Inc, Ashland, OR, USA).

\section{RNA extraction and quantitative real time polymerase chain reaction ( $\mathrm{QRT}-\mathrm{PCR}$ )}

Mouse J774 macrophages $\left(3 \times 10^{6}\right.$ cells/well $)$ were infected with live $C$. trachomatis $\left(3 \times 10^{5} \mathrm{IFU} /\right.$ well $)$ in 6 well plates for 48 hours followed by replacement of fresh media containing Ag-PVP nanoparticles. RNA was extracted from the cell pellets using the Qiagen RNeasy Kit (Qiagen Inc, Valencia, CA, USA), which included a DNase-I digestion step. qRT-PCR was employed to quantify mRNA gene transcripts of various inflammatory genes using the TaqMan ${ }^{\circledR}$ RNA-to- $\mathrm{C}_{\mathrm{T}}{ }^{\mathrm{TM}} 1$-step kit in combination with TaqMan ${ }^{\circledR}$ gene expression assays (Applied Biosystems-Life Technologies, Carlsbad, CA, USA) as reported..$^{38}$ Amplification of gene transcripts was performed according to the manufacturer's protocol using ABI ViiA ${ }^{\mathrm{TM}} 7$ real time PCR (Applied Biosystems) and standard amplification conditions. The relative changes in gene expression were calculated using the equation: $2^{-\triangle \Delta C T}$ where all values were normalized with respect to the "housekeeping" gene GAPDH (glyceraldehyde 3-phosphate dehydrogenase) mRNA levels. Amplification using $100 \mathrm{ng}$ RNA was performed in a volume of $20 \mu \mathrm{L}$. Each real time PCR assay was performed in triplicate and the results expressed as the mean \pm standard deviation.

\section{Statistical analysis}

The two-tailed unpaired Student's $t$-test was used to compare the data. $P<0.05$ was considered significant.

\section{Results}

\section{Cytotoxicity analysis of Ag-PVP nanoparticles}

Toxicity is the most pressing problem for metal-based nanoparticles in medicinal applications. Therefore, we tested the toxicity of all sizes of Ag-PVP nanoparticles to mouse J774 macrophages prior to investigating their potential as anti-inflammatory agents against a $C$. trachomatis infection. The MTT results revealed that concentrations of Ag-PVP nanoparticles above $25 \mu \mathrm{g} / \mathrm{mL}$ were extremely toxic to cells ( $<40 \%$ viable cells), whereas concentrations less than $6.25 \mu \mathrm{g} / \mathrm{mL}$ had greater cell viability ( $>80 \%$ viable cells) (Figure 1). We observed that Ag-PVP nanoparticles of $10 \mathrm{~nm}$ were less toxic to cells than the 20 and $80 \mathrm{~nm}$ sizes at similar concentrations ( $>90 \%$ viable cells) (Figure 1). Our MTT assay revealed a toxicity trend and higher cell viabilities in the order of $10 \mathrm{~nm}>20 \mathrm{~nm}>80 \mathrm{~nm}$ for all tested concentrations. Besides giving clues on the toxicity effect of different sizes of Ag-PVP nanoparticles, the MTT results also provided the desirable concentration range $(0.39-6.25 \mu \mathrm{g} / \mathrm{mL})$ for all subsequent experiments.

\section{All sizes of Ag-PVP nanoparticles reduced IL-6 and TNF elicited from macrophages infected with live $C$. trachomatis}

C. trachomatis infection of macrophages results in the secretion of several inflammatory mediators, amongst

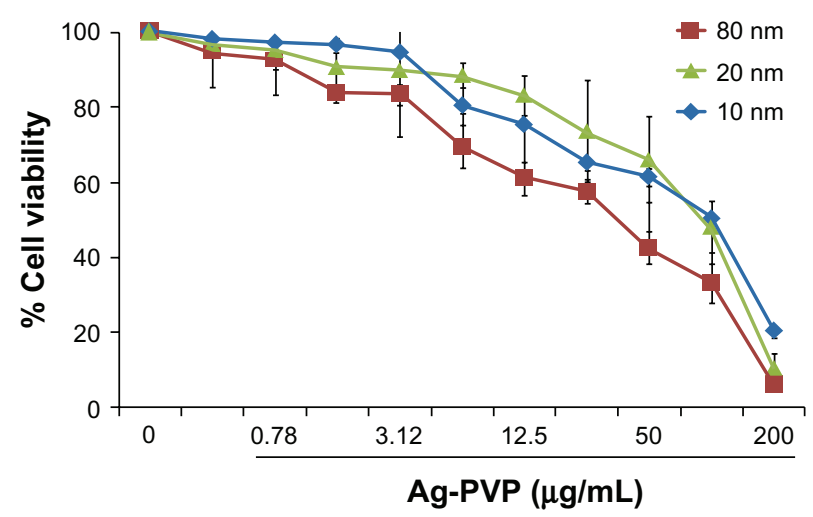

Figure I Silver-polyvinyl pyrrolidone (Ag-PVP) nanoparticle toxicity to mouse J774 macrophages is concentration dependent.

Notes: Mouse $\mathbf{7 7 4}$ macrophages were seeded in a 96 well plates at a density of $10^{5}$ cells/well/50 $\mu \mathrm{L}$ in the presence or absence of Ag-PVP nanoparticles in concentrations ranging from 0.39 to $200 \mu \mathrm{g} / \mathrm{mL}$. The Cell-Titer 96 cell Proliferation Assay kit was used to determine cell viability as described in the Materials and methods section. Absorbance was read at $570 \mathrm{~nm}$ and percent cell viability was calculated by using the optical density readings compared to normal cells. The data are representative of three separate experiments.

Abbreviations: Ag-PVP, silver-polyvinyl pyrrolidone; nm, nanometer. 
which are the cytokines IL- 6 and TNF. ${ }^{19}$ Hence, we first selected IL-6 and TNF as markers of inflammation to determine the anti-inflammatory effect of Ag-PVP nanoparticles and their secretion from C. trachomatis infected macrophages. Mouse $\mathbf{J} 774$ macrophages infected with live C. trachomatis showed marked infectivity that correlated with the significant secretion levels of IL-6 (Figure 2A) and TNF (Figure 2B) when compared to the levels from uninfected macrophages. Nevertheless, infected macrophages exposed to various concentrations of 10,20 and $80 \mathrm{~nm}$ sizes of Ag-PVP nanoparticles (0.39-6.25 $\mu \mathrm{g} /$ $\mathrm{mL}$ ) secreted less IL-6 (Figure 2A) and TNF (Figure 2B) in a dose dependent manner, especially at concentrations of 3.12 and $6.25 \mu \mathrm{g} / \mathrm{mL}$. Additional kinetic studies revealed that Ag-PVP nanoparticles $(10 \mathrm{~nm})$, when used at concentrations of 2.5 and $5 \mu \mathrm{g} / \mathrm{mL}$, were equally effective at reducing cytokine levels (data not shown). Evidently, Ag-PVP nanoparticles of the $10 \mathrm{~nm}$ size exhibited greater anti-inflammatory effect than the 20 and $80 \mathrm{~nm}$ sizes, and
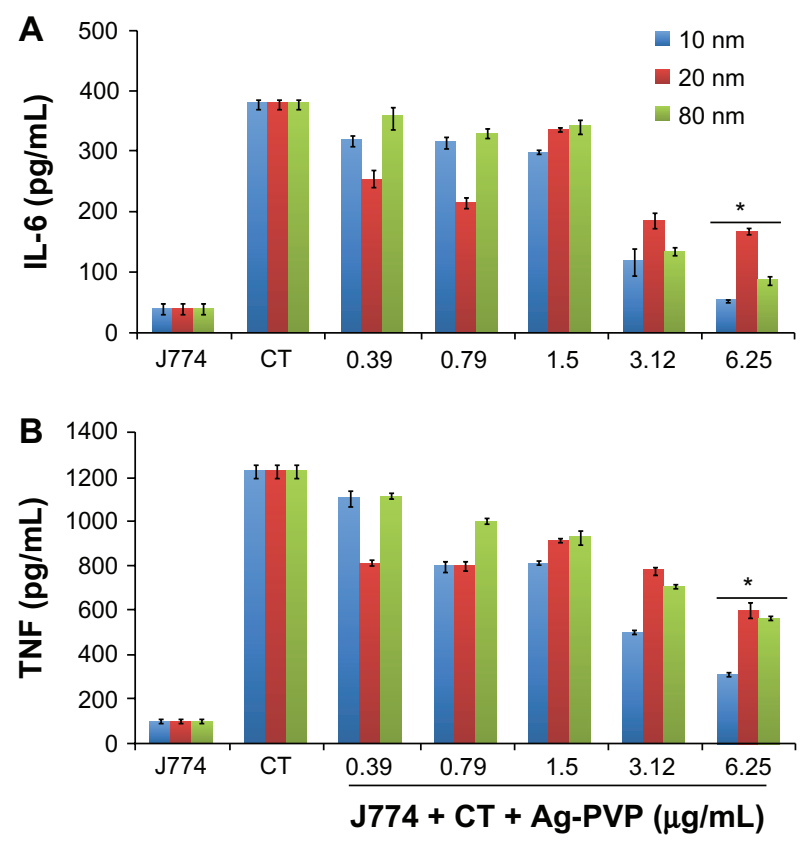

Figure 2 Silver-polyvinyl pyrrolidone (Ag-PVP) nanoparticles downregulate interleukin (IL)-6 and tumor necrosis factor (TNF) in C. trachomatis infected mouse j774 macrophages.

Notes: Mouse $\mathrm{J774}$ macrophages $\left(10^{6}\right.$ cells $/ \mathrm{mL}$ ) were seeded in 24 well plates and were either left uninfected or infected with live $C$. trachomatis $\left(10^{5}\right.$ inclusion forming units [IFU]/well). After 2 days post infection, Ag-PVP nanoparticles at 0.0 .39 to $6.25 \mu \mathrm{g} / \mathrm{mL}$ were added to cell cultures and the production levels of IL-6 (A) and TNF (B) were quantified in cell-free supernatants collected 48 hours later by ELISA. *indicates significant difference $(P<0.05)$ between live $C$. trachomatis and live $C$. trachomatis + Ag-PVP nanoparticles $(6.25 \mu \mathrm{g} / \mathrm{mL})$ and was calculated by using the twotailed unpaired Student's $t$-test. Each bar represents the mean \pm standard deviation of samples run in duplicate. The data are representative of two separate experiments. Abbreviations: Ag-PVP, silver-polyvinyl pyrrolidone; CT, live Chlamydia trachomatis; IL-6, interleukin-6; TNF, tumor necrosis factor; ELISA, enzyme-linked immunosorbent assay. was therefore employed for further studies. The significant anti-inflammatory effect in the concentration range of 2.5 to $6.25 \mu \mathrm{g} / \mathrm{mL}$ ruled out the possibility that Ag-PVP nanoparticle inhibition was due to cell death since these concentrations were not toxic to cells (Figure 1). These findings provide more evidence for the anti-inflammatory properties of Ag-PVP nanoparticles, and give insight to the potential of Ag-PVP nanoparticles in controlling excessive C. trachomatis inflammatory responses.

\section{Confocal microscopy analysis confirms the effect of Ag-PVP nanoparticles on IL-6 and TNF secretion in C. trachomatis infected macrophages}

Confocal microscopy was employed to provide physical evidence for the anti-inflammatory effect of Ag-PVP nanoparticles on the secretion levels of TNF and IL- 6 by C. trachomatis infected macrophages, and also to support our ELISA results. In comparison to uninfected macrophages, infected macrophages had greater green and red florescence aggregating around the nucleus indicating the presence of TNF and IL-6, respectively (Figure 3). Conversely, when infected macrophages were exposed to Ag-PVP nanoparticles of $10 \mathrm{~nm}$, TNF and IL-6 aggregation patterns were qualitatively lower (Figure 3), and thus corroborated our ELISA

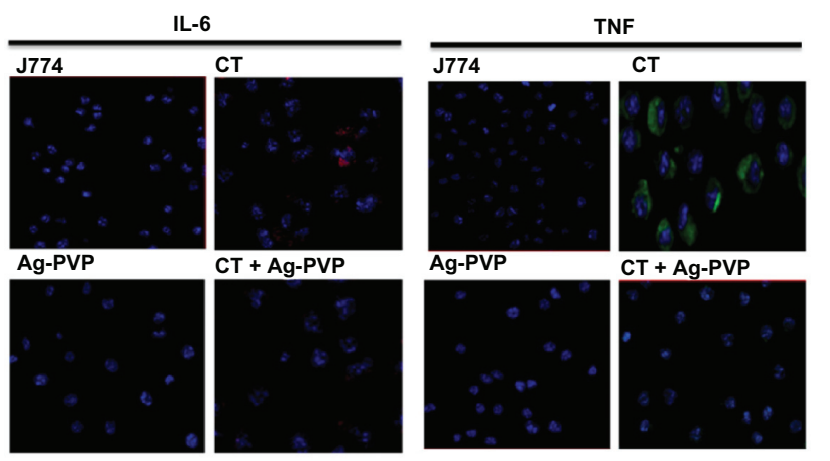

Figure 3 Microscopic analysis to confirm the effect of silver-polyvinyl pyrrolidone (Ag-PVP) nanoparticles on the secretion of tumor necrosis factor (TNF) and interleukin (IL)-6 in C. trachomatis infected macrophages.

Notes: Mouse $\mathrm{J774}$ macrophages $\left(2.5 \times 10^{4}\right.$ cells/well $)$ were left uninfected or infected with $C$. trachomatis $\left(2.5 \times 10^{3} \mathrm{IFU} /\right.$ well), and after 2 days infection, the media were replaced with fresh media supplemented with or without Ag-PVP nanoparticles $(2.5 \mu \mathrm{g} / \mathrm{mL})$. After 48 hours post Ag-PVP nanoparticle incubation, the cells were fixed with $2 \%$ paraformaldehyde and then immunostained with anti-mouse TNF (fluorescein isothiocyanate) and IL-6 (phycoerythrin) antibodies. The slides were stained with DAPI combined with an antifade mounting solution. Slides were examined using a confocal microscope (Nikon Eclipse Ti Confocal Microscope). Green (TNF) and red (IL-6) fluorescence aggregating around the nucleus (blue) indicates secretion of cytokines.

Abbreviations: Ag-PVP, silver-polyvinyl pyrrolidone; CT, live Chlamydia trachomatis; IL-6, interleukin-6; TNF, tumor necrosis factor; IFU, inclusion forming units; DAPI, 4',6-diamidino-2-phenylindole. 
observations of the anti-inflammatory effect of Ag-PVP nanoparticles.

\section{The effect of Ag-PVP nanoparticles on the production levels of inflammatory mediators in C. trachomatis infected j774 macrophages}

Given that $C$. trachomatis induces the secretion of TNF and IL-6 upon its infection of macrophages, we next investigated the effect of Ag-PVP nanoparticles on additional cytokines and chemokines using multiplex ELISA. Infected macrophages produced significant $(P<0.05)$ quantities of cytokines (IL-6, TNF, IL-12p70, IL-1 $\alpha$, IL-1 $\beta$, IL-9, IL-15, GM-CSF [granulocyte-macrophage colony stimulating factor], G-CSF [granulocyte colonystimulating factor]) (Figure 4A and B, Figure 5A and B) and chemokines (chemokine [C-X-C motif] ligand [CXCL] 10, CXCL1, CXCL5, chemokine [C-C motif] ligand [CCL] 5, CCL2) (Figure 4C and D, Figure 5C-E) when compared to macrophages exposed to Ag-PVP nanoparticles alone or to macrophages that remained uninfected. However, Ag-PVP nanoparticles, when added to $C$. trachomatis infected macrophages, significantly $(P<0.05)$ diminished the production levels of IL-12p70, GM-CSF, IL-1 $\alpha$, TNF, IL-6, G-CSF, CXCL10, and CCL5 (Figure 4A-D) but not those of IL-1 $\beta$, IL-9, IL-15, IL-5, IL-3, CCL2, CXCL1, and CXCL5 (Figure 5A-E). Collectively, our results indicate that Ag-PVP nanoparticles have an anti-inflammatory effect on selected cytokines and chemokines triggered by C. trachomatis in macrophages.

\section{The effect of Ag-PVP nanoparticles on the mRNA gene transcript expression of TLR2 and NOD2}

Mammalian cells sense the presence of invading microbial pathogens through recognition of PAMPs, which include receptors like NOD and TLR. ${ }^{39}$ Since C. trachomatis induced the release of early inflammatory mediators, ${ }^{40}$ we next investigated the possibility that Ag-PVP nanoparticles altered early receptor expression of NOD and TLR to reduce the production levels of cytokines triggered by $C$. trachomatis infected macrophages. We showed by qRT-PCR that $C$. trachomatis induced a 2.2 and 2.8 fold upregulation in the expression of the mRNA gene transcripts for TLR2 and NOD2 (Figure 6A) of macrophages, respectively, in comparison to uninfected macrophages. However, in the presence of added Ag-PVP
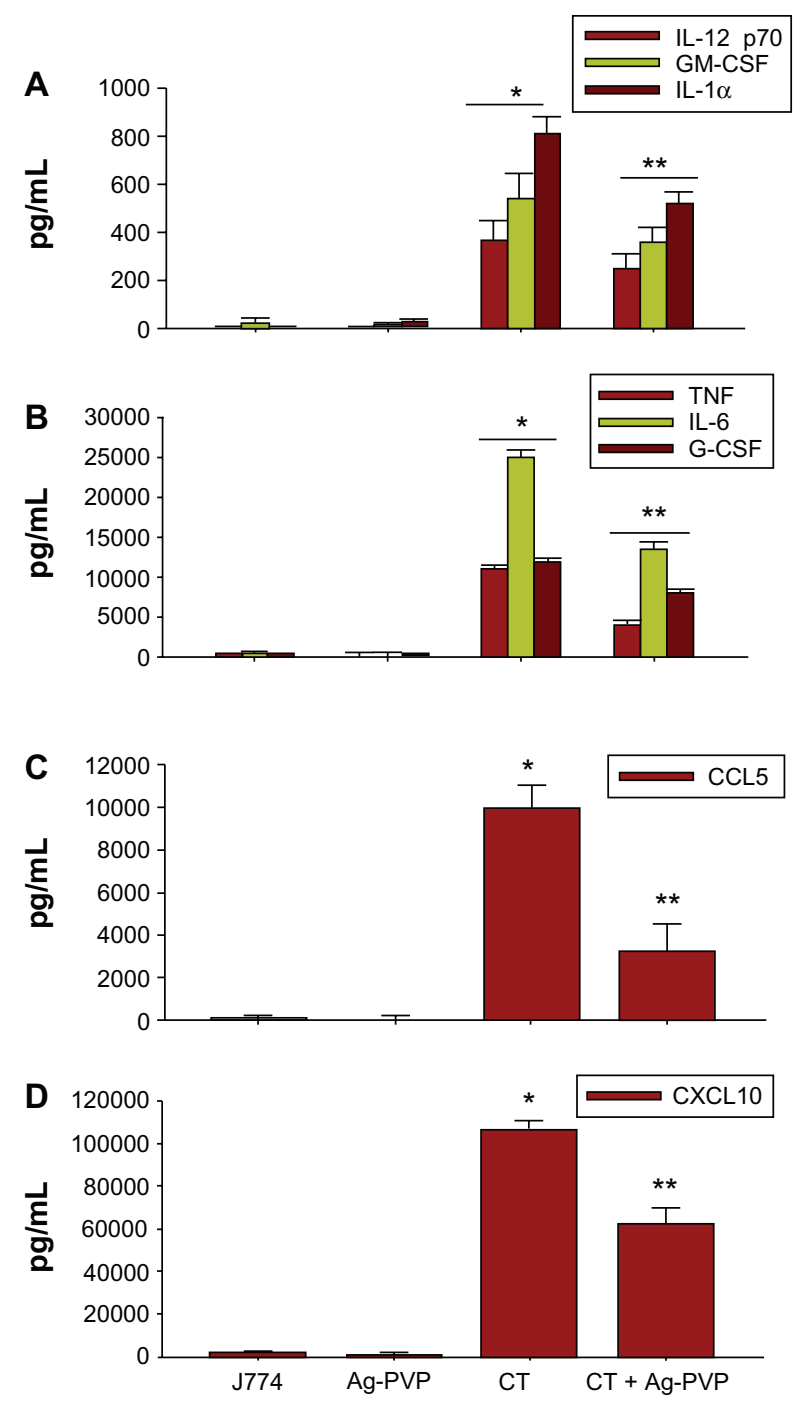

Figure 4 Silver-polyvinyl pyrrolidone (Ag-PVP) nanoparticles downregulate the production level of various inflammatory mediators in C. trachomatis infected macrophages.

Notes: Mouse $\mathrm{J} 774$ macrophages $\left(10^{6}\right.$ cells $\left./ \mathrm{mL}\right)$ were seeded in 24 well plates and were either left uninfected or infected with live $C$. trachomatis $\left(10^{5} \mathrm{IFU} / \mathrm{mL}\right)$. After 2 days infection, Ag-PVP nanoparticles $(2.5 \mu \mathrm{g} / \mathrm{mL})$ were added to cell cultures and the production levels of various cytokines $(\mathbf{A}$ and $\mathbf{B})$ and chemokines $(\mathbf{C}$ and $\mathbf{D})$ were quantified in supernatants collected 2 days later by multiplex ELISA. $* P<0.05$ is considered significant when compared between $\mathrm{J74}$ and live $C$. trachomatis; $* * P<0.05$ is considered significant when compared between live $C$. trachomatis and live $C$. trachomatis + Ag-PVP nanoparticles. Each bar represents the mean \pm standard deviation of samples run in duplicate.

Abbreviations: Ag-PVP, silver-polyvinyl pyrrolidone; CCL5, chemokine (C-C motif) ligand 5; CXCLI0, chemokine (C-X-C motif) ligand 10; CT, live Chlamydia trachomatis; IL, interleukin; G-CSF, granulocyte-colony stimulating factor; GM-CSF, granulocyte-macrophage colony stimulating factor; TNF, tumor necrosis factor; ELISA, enzyme-linked immunosorbent assay.

nanoparticles, the mRNA expression levels of NOD2 and TLR2 were reduced (approximately 2 fold reduction) (Figure 6A). Therefore, the ability of Ag-PVP nanoparticles to reduce the expression levels of these receptors strongly suggest potential mechanism(s) by which Ag-PVP nanoparticles may regulate inflammatory mediators triggered by $C$. trachomatis in macrophages. 

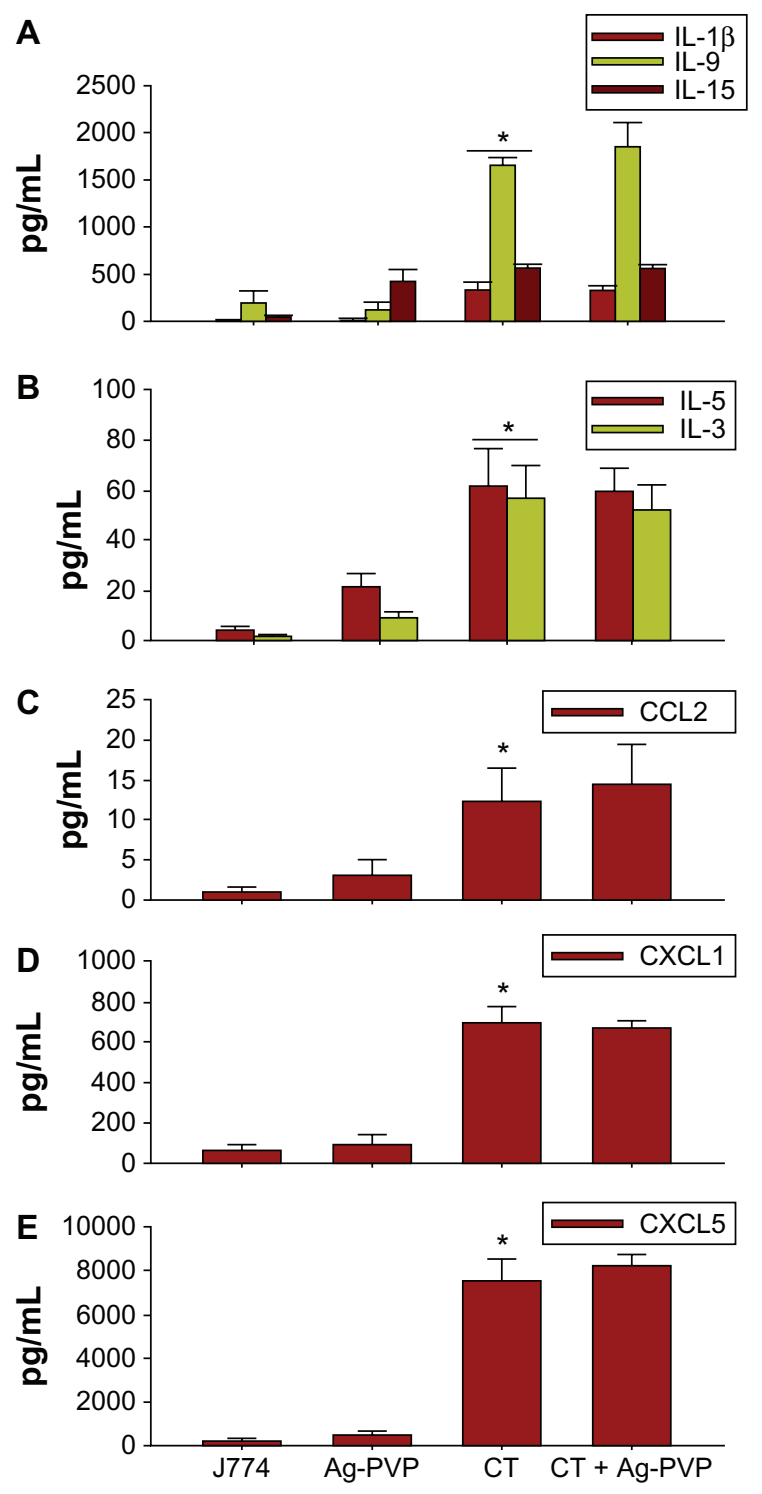

Figure 5 Failure of silver-polyvinyl pyrrolidone (Ag-PVP) nanoparticles to downregulate selected inflammatory mediators in $C$. trachomatis infected macrophages.

Notes: Mouse 1774 macrophages were stimulated and inflammatory mediators quantified as described above in Figure 4. Shown are the production levels of cytokines $(\mathbf{A}$ and $\mathbf{B})$ and chemokines $(\mathbf{C}-\mathbf{E}) . P<0.05$ is considered significant when compared between $\mathrm{J774}$ and live $C$. trachomatis as determined by the two-tailed unpaired Student's $t$-test. Each bar represents the mean \pm standard deviation of samples run in duplicate. The data are representative of two separate experiments. Abbreviations: Ag-PVP, silver-polyvinyl pyrrolidone; CCL2, chemokine (C-C motif) ligand 2; CT, live Chlamydia trachomatis; CXCL, C-X-C motif chemokine ligand; IL, interleukin.

\section{Ag-PVP downregulates the mRNA gene transcript expression of interleukin-I receptor associated kinase (IRAK) 3, CD40, and matrix metallopeptidase (MMP) 9}

Like pattern recognition receptors, perturbations in inflammatory signaling pathways may affect the secretion of
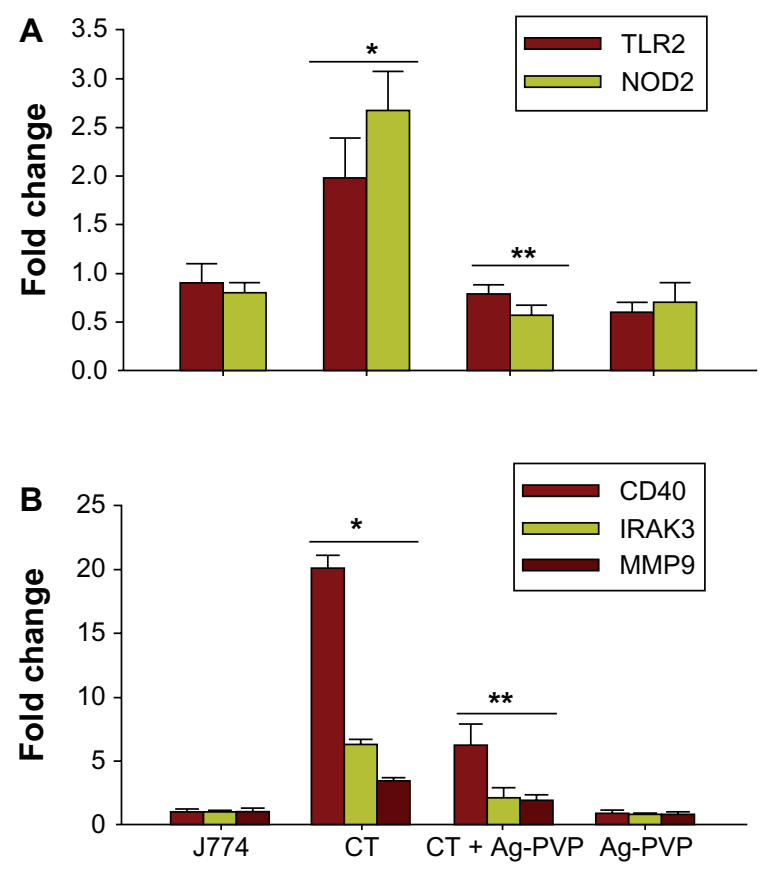

Figure 6 Silver-polyvinyl pyrrolidone (Ag-PVP) nanoparticles reduce the mRNA transcription of toll like receptor 2 (TLR2), nucleotide-binding oligomerization domain-containing protein-2 (NOD2), interleukin-I receptor associated kinases 3 (IRAK3), CD40, and matrix metallopeptidase 9 (MMP9) by C. trachomatis in mouse J774 macrophages.

Notes: Macrophages $\left(3 \times 10^{6}\right.$ cells $\left./ \mathrm{mL}\right)$ were left uninfected or infected with live $C$. trachomatis $\left(3 \times 10^{5}\right.$ IFU/well). After 2 days infection, Ag-PVP nanoparticles $(2.5 \mu \mathrm{g} /$ $\mathrm{mL}$ ) were added to macrophage cultures and 2 days later total RNA was extracted. One step qRT-PCR was used to quantify the mRNA gene transcripts of TLR2 and NOD2 (A) and IRAK3, CD40, and MMP9 (B). Data shown is an average of triplicate runs. ${ }^{*} P<0.05$ is considered significant when compared between $J 774$ and live $C$. trachomatis and $* * P<0.05$ is considered significant when compared between live C. trachomatis and live $C$. trachomatis + Ag-PVP nanoparticles as determined by the two-tailed unpaired Student's $t$-test.

Abbreviations: Ag-PVP, silver-polyvinyl pyrrolidone; CT, live Chlamydia trachomatis; IRAK3, interleukin-I receptor associated kinases 3; MMP9, matrix metallopeptidase 9; NOD2, nucleotide-binding oligomerization domain-containing protein-2; TLR2, toll like receptor 2; $C D$, cluster of differentiation; qRT-PCR, quantitative real time polymerase chain reaction; mRNA, messenger ribonucleic acid.

cytokines and chemokines from C. trachomatis infected macrophages. Genes encoding mediators from the Toll/ IL receptor immune signal transduction pathway mediate signaling from TLR to specific interleukin receptor family members, such as IRAKs, which maintains stable binding between the cytokine and its corresponding receptors. Our qRT-PCR findings clearly revealed upregulation of the mRNA expression of IRAK3 in C. trachomatis infected macrophages when compared with the expression level in uninfected macrophages (Figure 6B). However, when AgPVP nanoparticles were added to infected macrophages, the mRNA expression level of IRAK3 was markedly reduced (approximate 4 fold reduction) (Figure 6B).

Since the CD40 membrane protein has a role in transmitting inflammatory signaling for cytokine secretion, ${ }^{41}$ we also determined the effect of Ag-PVP nanoparticles on the 
expression of its mRNA gene transcript levels. By qRT-PCR, we showed upregulation (20 fold increase) in the expression of CD40 at the mRNA gene transcript level in C. trachomatis infected macrophages when compared with uninfected macrophages (Figure 6B). Alternatively, the mRNA expression level of CD40 was reduced significantly (approximate 15 fold reduction) in the presence of Ag-PVP nanoparticles (Figure 6B). Taken together, the ability of Ag-PVP nanoparticles to reduce the expression levels of both IRAK3 and CD40 indicates its capacity to perturb genes encoding mediators of inflammatory signaling pathways, and to possibly further reduce inflammatory mediators in C. trachomatis infected macrophages.

The development of inflammation arising from infection with $C$. trachomatis is mainly due to the host immune response generated against eradicating the pathogen. ${ }^{13-16}$ MMPs are involved in the breakdown of extracellular matrix and thus play important roles in inflammation. We investigated whether Ag-PVP nanoparticles perturbed the expression of MMPs. Of the many MMPs involved in promoting inflammation, we focused on MMP9 because neutrophils, which are rapidly recruited during a Chlamydia infection, release MMP9 to contribute to tissue damage. ${ }^{42}$ We showed by qRT-PCR a 2.5 fold increase in the level of the mRNA gene transcript expression of MMP9 in C. trachomatis infected macrophages when compared with uninfected macrophages, suggesting enhancement of MMP9 during early infection (Figure 6B). When Ag-PVP nanoparticles were added to infected macrophage cultures, the mRNA expression level of MMP9 reduced significantly (approximate 2 fold reduction) (Figure 6B), suggesting the inhibitory effect of Ag-PVP nanoparticles on MMP9 expression that additionally curtails the inflammatory process.

\section{Ag-PVP nanoparticles reduced the expression of CD80 and CD86}

Pathogens are recognized during host pathogen interactions via pattern recognition receptors, including Toll and NOD, to trigger the production of inflammatory mediators for initiating innate immune responses and the expression of costimulatory molecules for subsequent adaptive immune responses. To broaden our understanding of Ag-PVP nanoparticles on host immune responses, we tested its effect on regulating CD80 and CD86 costimulatory molecules, at both the protein and mRNA gene transcript levels, in macrophages infected with live C. trachomatis. Flow cytometric analyses revealed protein expression changes for CD86 (Figure 7A) and CD80 (Figure 7B) in C. trachomatis infected mouse J774 macrophages as exemplified by the peak shifts in
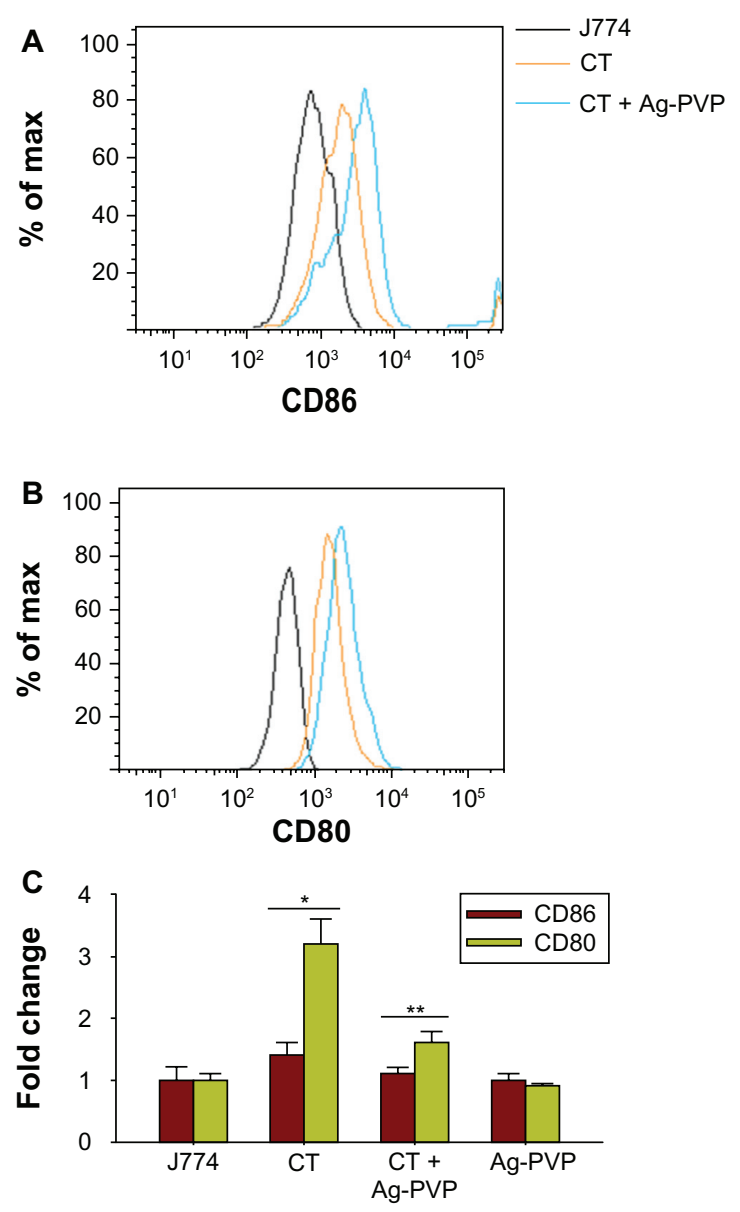

Figure 7 Silver-polyvinyl pyrrolidone (Ag-PVP) nanoparticles downregulate the expression levels of CD80 and CD86 in C. trachomatis infected mouse $\mathrm{J} 774$ macrophages at both the protein and gene level.

Notes: Mouse $\mathrm{J774}$ macrophages were cultured and infected as described in the Materials and methods section for flow cytometry and qRT-PCR experiments. After 2 days of infection, $2.5 \mu \mathrm{g} / \mathrm{mL}$ Ag-PVP nanoparticles were added to cell culture, and 2 days later, samples were analyzed by flow cytometry for protein expression and qRT-PCR for mRNA expression. Shown are the expression shifts of CD86 (A) and CD80 (B) at the protein and gene levels (C). ${ }^{* P}<0.05$ is considered significant when compared between $\mathrm{J774}$ and live $C$. trachomatis and $* * P<0.05$ is considered significant when compared between live $C$. trachomatis and live $C$. trachomatis $+\mathrm{Ag}-\mathrm{PVP}$ nanoparticles as determined by the two-tailed unpaired Student's $t$-test.

Abbreviations: Ag-PVP, silver-polyvinyl pyrrolidone; CT, live Chlamydia trachomatis; $C D$, cluster of differentiation; $q R T-P C R$, quantitative real time polymerase chain reaction; $m R N A$, messenger ribonucleic acid.

their respective fluorescence intensities when compared to uninfected macrophages. Conversely, in the presence of added Ag-PVP nanoparticles, the peak shifted toward that of the uninfected macrophages, indicating a reduction in the expression of CD86 and CD80 by Ag-PVP nanoparticles (Figure 7A and B), and in particular for CD86.

Additionally, we demonstrated by qRT-PCR a 1.5 and 3.2 fold increase in the mRNA gene transcript expression for CD86 and CD80, respectively, in C. trachomatis infected macrophages when compared with uninfected macrophages, suggesting moderate enhancement of CD80 and CD86 during infection of macrophages, especially for CD80 (Figure 7C). 
Again, the inhibitory effect of Ag-PVP nanoparticles was observed by its ability to reduce CD 86 , and especially CD80, mRNA expression levels in infected macrophages (Figure 7C). In general, our results from both the flow cytometry and qRT-PCR studies gave clues on the role of Ag-PVP nanoparticles in regulating adaptive immune responses to potentially control C. trachomatis inflammation.

\section{Discussion}

The interaction of microbes with host cells encompasses two major events causing either disease or establishment of a long-term relationship with the host. ${ }^{13}$ Some pathogens manage the long-term relationship by eliciting chronic immune responses through misdirecting the normal host immune responses to cause immunopathologies as reported for C. trachomatis. ${ }^{13,14}$ Inflammation of an infected tissue has several beneficial effects in combating infection by recruiting cells and molecules of innate immunity to destroy the pathogen, as well as by activating lymphocytes to initiate adaptive immune responses. Continuous production of inflammatory mediators, as evidenced during a Chlamydia infection, lead to chronic inflammation and tissue damage, tubal infertility, and ectopic pregnancies, which are key characteristics of disease pathogenesis. ${ }^{13,19,43,44}$

Silver has been shown to have both antimicrobial and anti-inflammatory properties. ${ }^{23-27}$ Despite the many benefits of nanotechnology in biomedical applications, studies indicate that certain nanoparticles cause adverse effects because of their indiscriminate entry into various organs. ${ }^{33}$ In our study, more than $80 \%$ of cells remained viable at Ag-PVP nanoparticle concentrations ranging from $0.39-3.12 \mu \mathrm{g} / \mathrm{mL}$ (Figure 1), suggesting that these concentrations are not toxic to cells. Worthy of mention is that we used lower concentrations of silver nanoparticles in our study than what has been reported to have antimicrobial effects against viruses and bacteria. ${ }^{29-32}$ Also, concentrations above $25 \mu \mathrm{g} / \mathrm{mL}$ were highly toxic to macrophages, thus corroborating previous in vitro toxicity studies of various silver nanoparticles to mammalian cells. ${ }^{45} \mathrm{We}$ also observed that Ag-PVP nanoparticles of $10 \mathrm{~nm}$ were less toxic than the larger sizes (Figure 1). One plausible explanation for cytotoxicity differences in the three different sizes used in our study may be attributed to the notion that small sizes of Ag-PVP nanoparticles have high mobility and do not aggregate in one location as occurs with the larger sizes.

In the burn wound model, as well as in a peritoneal adhesion model in mice, silver nanoparticles significantly lowered the levels of IL-6. ${ }^{35,37}$ We report here that all sizes of tested
Ag-PVP nanoparticles $(10,20$, and $80 \mathrm{~nm})$ significantly reduced the levels of IL-6 and TNF (Figure 2). The production of IL- 6 and TNF is recognized as an initiator of events in the physiological alterations of inflammation to recruit and activate immune cells, such as neutrophils and macrophages, to the site of infection. ${ }^{46}$ The ability of all three sizes of AgPVP nanoparticles to downregulate IL- 6 and TNF suggests the applicability of Ag-PVP nanoparticles at broader size ranges, thus making it a potent anti-inflammatory molecule.

Ag-PVP nanoparticles not only regulated IL-6 and TNF levels, but also regulated a plethora of other cytokines and chemokines such as IL-12 p70, GM-CSF, IL-1 $\alpha$, G-CSF, CXCL10, and CCL5 (Figure 4) which can be viewed as being beneficial to the host in controlling excessive inflammatory mediators triggered by a C. trachomatis infection. Decreasing the released levels of these inflammatory mediators subsequently lowers cellular proliferation and extracellular matrix production, and thus, there is less inflammation. At the same time, other inflammatory mediators such as IL-1 $\beta$, IL-9, IL-15, IL-5, IL-3, CXCL5, CXCL1, and CCL2 were not adversely affected by Ag-PVP nanoparticles (Figure 5), suggesting that the anti-inflammatory effect of Ag-PVP nanoparticles is not a generalized phenomenon but rather one that is highly selective, and that may depend on the pathogen or cell type that it is exposed to. This revealing anti-inflammatory property of Ag-PVP nanoparticles permits it to be explored for a variety of disease settings as demonstrated here for our C. trachomatis in vitro model system. Even though the antiinflammatory effect of silver nanoparticles has been confirmed in various infection models, ${ }^{37,47-49}$ to our knowledge, this is the first study to report on its anti-inflammatory effect in a C. trachomatis infection model.

The inflammatory process is a complex phenomenon induced by different pathways and therefore many hypothetical possibilities can be explored to explain the anti-inflammatory mode of action of Ag-PVP nanoparticles. We have shown in our study that the anti-inflammatory effect of Ag-PVP nanoparticles was not attributed to apoptosis of inflammatory cells (Figure 1) and alternatively, that it may be due to perturbations in inflammatory signaling pathways. In this study, we observed that TLR2 and NOD2 mRNA expression levels were upregulated in C. trachomatis infected macrophages (Figure 6A), which is in agreement with previous findings where epithelial cells infected with C. muridarum had increased expression of NOD2 and TLR2. ${ }^{39}$ Similarly, NOD2 plays a major role in recognizing microbial products of gram-negative bacteria ${ }^{9-12}$ with $C$. trachomatis being no exception as it upregulated NOD2 in macrophages, as reported here. Both TLRs and NOD 
like receptors induce inflammatory signaling pathways for the secretion of inflammatory mediators. Thus, the capacity of Ag-PVP nanoparticles to reduce the expression of TLR2 and NOD2 infers less interaction between macrophages and C. trachomatis, and thereby less binding to its cognate receptors to trigger secretion of inflammatory mediators.

Additional receptors and downstream signaling events are equally vital for the secretion of inflammatory mediators. Here, we have focused on two such molecules: IRAK3 which inhibits the dissociation of IRAK 1 and IRAK4 from the TLR signaling complex, and CD40, a member of the TNF receptor superfamily that is essential in mediating a variety of immune and inflammatory responses. ${ }^{41}$ Both IRAK3 and CD40 were induced by $C$. trachomatis infected mouse macrophages, suggesting the importance of these signaling events during C. trachomatis inflammation (Figure 6B). That AgPVP nanoparticles reduced both CD40 and IRAK3 mRNA expression underscores that it may exert its anti-inflammatory effect partially by interfering with TNF and IL-1 receptors. Results from an in vitro study indicate that silver nanoparticles inhibit the interaction between glycoprotein 120 and CD4 to inhibit human immunodeficiency virus-1 infection. ${ }^{50}$ Although we have not directly demonstrated the interaction of Ag-PVP nanoparticles with $C$. trachomatis infected macrophages, it can be speculated that inhibition of CD40 and IRAK3 by Ag-PVP nanoparticles may interfere with C. trachomatis binding to macrophages, and ultimately lower the levels of secreted inflammatory mediators. Other reported mechanisms for the anti-inflammatory actions of silver nanoparticles include regulation of NF-кВ transcription in allergic airway inflammation, ${ }^{47}$ and regulation of the VEGF signaling pathway in allergic airway inflammation, ${ }^{48}$ or in individuals with asthma. ${ }^{51}$ In our study, the capacity of Ag-PVP nanoparticles to reduce TLR2, NOD2, CD40, and IRAK3 levels suggest mechanisms subsequently leading to potential downregulation of $\mathrm{NF}-\mathrm{\kappa B}$, and therefore lower levels of inflammatory mediators.

For any infectious disease situation, the acute inflammatory state normally represents immediate modification of the extracellular matrix which involves MMPs and other proteolytic enzymes to initiate remodeling and tissue regeneration of the affected tissue. However, as the infection persists, the presence of MMPs permit the influx of inflammatory cells with tissue damage ensuing. Therefore, persisting or excessive inflammation can indicate overexpression of MMPs, enhanced repair responses, and extracellular matrix accumulation and fibrosis. Infection in the murine model of Chlamydia urogenital infection induced excessive extracellular matrix modification with lumen occluding fibrosis, which correlated with hydrosalpinx formation and infertility. ${ }^{42}$ These findings, as well as MMP expression in trachoma, ${ }^{31}$ and the in vitro expression of MMPs in response to $C$. trachomatis infection in human fallopian tube organ culture, highlights the significance of MMPs in C. trachomatis pathogenesis. ${ }^{13,42}$ We observed in our study that MMP9 expression was upregulated by $C$. trachomatis infected macrophages as expected, but downregulated by Ag-PVP nanoparticles (Figure 6B). Interpretation of our results can be complex since we only tested one form of MMP. However, the ability of Ag-PVP nanoparticles to reduce MMP9 is of major significance as excessive secretion of MMP9 may contribute to the pathogenesis of tissue destruction in a wide array of diseases, including Chlamydia. ${ }^{42}$ Furthermore, MMP9 contributes to tissue destruction and inflammation via degradation of matrix proteins and proteolytic activation of cytokines/chemokines during C. trachomatis infections. ${ }^{13,14,42}$

The expression of CD80 and CD86, along with major histocompatibility class molecules, allows the presentation of microbial peptides to activate naïve CD4 T cells to produce pro-inflammatory mediators at the site of infection. CD80 and CD86 reportedly regulate innate immune responses in murine polymicrobial sepsis, which is a systemic inflammatory response to infection. ${ }^{52} \mathrm{CD} 80$ and $\mathrm{CD} 86$ are stimulated via CD28, while cytotoxic T lymphocyte antigen 4 serves as both a stoichiometric inhibitor of CD28-CD80/86 engagement, and directly induces immunosuppressive signals within dendritic cells. ${ }^{53-55}$ Indeed, the CD28-CD80/86 system is known to regulate inflammation in autoimmune diseases, where CD80/86 signals via NF- $\kappa B$ to induce numerous cytokines, most notable IL-6. ${ }^{53}$ In vivo, deletion or blockade of CD80/86 improves survival and attenuates pro-inflammatory cytokine production in CLP. ${ }^{56}$ Here, we have revealed that Ag-PVP nanoparticles reduced the expression of CD80 and CD86, both at the gene and protein levels in C. trachomatis infected macrophages (Figure 7), suggesting that Ag-PVP nanoparticles have the potential to act not only on cytokines produced by innate immune cells, but also those produced by $C$. trachomatis activated $\mathrm{T}$ cells during the adaptive immune response.

\section{Conclusion}

The development of inflammation arising from infection with $C$. trachomatis may depend on several factors including C. trachomatis virulence, the number of bacteria in the affected tissues, and the host immune response generated against the bacteria. The advancement in nanotechnology 
has enabled us to utilize particles in the nanoscale range and has created new therapeutic perspectives. In the case of silver nanoparticles, the available data reveal their potential as anti-inflammatory agents in a variety of infectious disease models and we are now including $C$. trachomatis to the list. Collectively, our findings demonstrate that Ag-PVP nanoparticles may mediate its anti-inflammatory effect in macrophages infected with $C$. trachomatis by regulating various upstream surface receptors and downstream inflammatory pathway genes. Our study provides further evidence on the anti-inflammatory properties of Ag-PVP nanoparticles and opens new possibilities for small sizes of Ag-PVP nanoparticles to be employed as regulators of inflammatory responses induced by C. trachomatis infection.

\section{Acknowledgments}

The project described was supported by NSF-CREST (HRD1241701) and NSF-HBCU-UP (HRD-1135863). We would like to thank Yvonne Williams and Lashaundria Lucas for their excellent administrative assistance.

\section{Disclosure}

The authors report no conflicts of interest in this work.

\section{References}

1. Stephens RS. Challenge of Chlamydia research. Infect Agent Dis. 1993;1:279-293.

2. Bavoil PM, Hsia RC, Rank RG. Prospects for a vaccine against Chlamydia genital disease: microbiology and pathogenesis. Bull Inst Pasteur. 1996;94:50-54.

3. Schachter J, Dawson CR. The epidemiology of trachoma predicts more blindness in the future. Scand J Infect Dis. 1990;69:55-62.

4. Joseph TD, Bose SK. A heat labile protein of Chlamydia trachomatis binds to HeLa cells and inhibits the adherence of chlamydiae. Proc Nat Acad Sci U S A. 1991;88:4054-4058.

5. Swanson AF, Kuo CC. Binding of the glycan of the major outer membrane protein of Chlamydia trachomatis to HeLa cells. Infect Immun. 1994;62:24-28.

6. Ting LM, Hsia RC, Haidaris CG, Bavoil PM. Interaction of outer envelope proteins of Chlamydia psittaci GPIC with the HeLa cell surface. Infect Immun. 1995;63:3600-3608.

7. Su H, Raymond L, Rockey D, Fischer E, Hackstadt T, Aldwell HD. A recombinant Chlamydia trachomatis major outer membrane. Infect Immun. 1996;65:11134-11140.

8. Raulston JE, Paul TR, Knight ST, Wyrick PB. Localization of Chlamydia trachomatis heat shock proteins 60 and 70 during infection of a human endometrial epithelial cell line in vitro. Infect Immun. 1998;66:2323-2329.

9. Chamaillard M, Hashimoto M, Horie Y, et al. An essential role for NOD1 in host recognition of bacterial peptidoglycan containing diaminopimelic acid. Nat Immunol. 2002;4:702-707.

10. Girardin SE, Boneca IG, Carneiro LA, et al. Nod1 detects a unique muropeptide from gram-negative bacterial peptidoglycan. Science. 2003;300:1584-1587.

11. Inohara N, Ogura Y, Fontalba A, et al. Host recognition of bacterial muramyl dipeptide mediated through NOD2: implications for Crohn's disease. J Biol Chem. 2003;278:5509-5512.
12. Girardin SE, Boneca IG, Viala J, et al. Nod2 is a general sensor of peptidoglycan through muramyl dipeptide (MDP) detection. $J$ Biol Chem. 2003;278:8869-8872.

13. Stephens RS. The cellular paradigm of chlamydial pathogensis. Trends Microbiol. 2003;11:44-51.

14. Kuo CC. Host response. In: Barron AL, editor. Microbiology of Chlamydia. Boca Raton: CRC press; 1988:193-208.

15. Taylor HR. An animal model of trachoma II. The importance of repeated re-infection. Invest Ophthalmol Vis Sci. 1982;23:507-515.

16. Patton DL, Taylor HR. The histopathology of experimental trachoma: ultrastructural changes in the conjunctival epithelium. J Infect Dis. 1986;153:870-878.

17. Skilton RJ, Cutcliffe LT, Barlow D, et al. Penicillin induced persistence in Chlamydia trachomatis: high quality time lapse video analysis of the development cycle. PLoS One. 2009;4:e7723-e7723.

18. Bain DL. Chlamydial genovar distribution after community wide antibiotic treatment. J Infect Dis. 2001;184:1581-1588.

19. Yilma AN, Singh SR, Fairley SJ, Taha MA, Dennis VA. The antiinflammatory cytokine, interleukin-10, inhibits inflammatory mediators in human epithelial cells and mouse macrophages to live and UV-inactivated Chlamydia trachomatis. Mediators Inflamm. 2012;2012:520174.

20. Byrne GI, Faubion CL. Lymphokine-mediated inhibition of Chlamydia replication in mouse fibroblasts is neutralized by anti-gamma interferon. Infect Immun. 1983;42:1152-1158.

21. Beatty WL, Morrison RP, Byrne GI. Reactivation of persistent Chlamydia trachomatis infection in cell culture. Infect Immun. 1995;63: 199-205.

22. Hoyme UB. Clinical significance of Crede's prophylaxis in Germany at present. Infect Dis Obstet Gynecol. 1993;1:32-36.

23. Kim JS, Kuk E, Yu KN, et al. Antimicrobial effects of silver nanoparticles. Nanomedicine. 2007;3:95-101.

24. Lok CN, Ho CM, Chen R, et al. Proteomic analysis of the mode of antibacterial action of silver nanoparticles. J Prot Res. 2006;5:916-924.

25. Morones JR, Elechiguerra JL, Camacho A, et al. The bactericidal effect of silver nanoparticles. Nanotechnology. 2005;16:2346-2353.

26. Shahverdi AR, Fakhimi A, Shahverdi HR, Minaian S. Synthesis and effect of silver nanoparticles on the antibacterial activity of different antibiotics against Staphylococcus aureus and Escherichia coli. Nanomedicine. 2007;3:168-171.

27. Sondi I, Salopek-Sondi B. Silver nanoparticles as antimicrobial agent: a case study on E. coli as a model for Gram-negative bacteria. J Colloid Interface Sci. 2004;275:177-182.

28. Sun RW, Rong C, Chung NPY, Ho CM, Lin CLS, Che CM. Silver nanoparticles fabricated in Hepes buffer exhibit cytoprotective activities toward HIV-1 infected cells. Chem Commun. 2005;1:5059-5061.

29. Lu L, Sun RW, Chen R, et al. Silver nanoparticles inhibit hepatitis B virus replication. Antivir Ther. 2008;13:253-262.

30. Sun L, Singh AK, Vig K, Pillai SR, Singh SR. Silver nanoparticles inhibit replication of respiratory syncytial virus. J Biomed Biotechnol. 2008;4:149-158.

31. Baram-Pinto D, Shukla S, Perkas N, Gedanken A, Sarid R. Inhibition of herpes simplex virus Type 1 infection by silver nanoparticles capped with mercaptoethane sulfonate. Bioconjugate Chem. 2009;20:1497-1502.

32. Rogers JV, Parkinson CV, Choi YW, Speshock JL, Hussain SM. A preliminary assessment of silver nanoparticle inhibition of monkeypox virus plaque formation. Nanoscale Res Lett. 2008;3:129-133.

33. Arora S, Jain J, Rajwade JM, Paknikar KM. Cellular responses induced by silver nanoparticles: in vitro studies. Toxicol Lett. 2008;179:93-100.

34. Kumari A, Kumar P, Ajayan PM, John G. Silver-nanoparticle-embedded antimicrobial paints based on vegetable oil. Nat Mater. 2008;7: 236-241.

35. Tain J, Wong KK, Ho CM, et al. Topical delivery of silver nanoparticles promotes wound healing. Chem Med Chem. 2007;2:129-136.

36. Sibbald RG, Contreras-Ruiz J, Coutts P, Fierheller J, Rothman A, Woo K. Bacteriology, inflammation, and healing: a study of nanocrystalline silver dressings in chronic venous leg ulcers. Adv Skin Wound Care. 2007;20:549-558. 
37. Wong KK, Cheung SO, Huang L, et al. Further evidence of the anti-inflammatory effects of silver nanoparticles. Chem Med Chem. 2009;4:1129-1135.

38. Gautam A, Dixit S, Philipp MT, et al. Interleukin-10 alters effect functions of multiple genes induced by Borrelia burgdorferi in macrophages to regulate Lyme disease inflammation. Infect Immun. 2011;79: 4876-4892.

39. Derbigny WA, Kerr MS, Johnson RM. Pattern recognition molecules activated by Chlamydia muridarum infection of cloned murine oviduct epithelial cell lines. J Immunol. 2005;175:6065-6075.

40. Johnson RM. Murine oviduct epithelial cell cytokine responses to Chlamydia muridarum infection include interleukin-12-p70 secretion. Infect Immun. 2004;72:3951-3960.

41. Gold JA, Parsey M, Hoshino Y, Hoshino S, Nolan A. CD40 contributes to lethality in acute sepsis: in vivo role for CD40 in innate immunity. Infect Immun. 2003;71:3521-3528.

42. Ramsey KH, Sigar IM, Schripsema JH, Shaba N, Cohoon KP. Expression of matrix metalloproteinases subsequent to urogenital Chlamydia muridarum infection of mice. Infect Immun. 2005;73:6962-6973.

43. Grayston JT, Wang SP, Yeh LJ, Kuo CC. Importance of reinfection in the pathogenesis of trachoma. Rev Infect Dis. 1985;7:717-725.

44. Molestina RE, Miller RD, Ramirez JA, Summersquill JT. Infection of human endothelial cells with Chlamydia pneumoniae stimulates transendothelial migration of neutrophils and monocytes. Infect Immun. 1999;67:1323-1330.

45. Ahamed M, AlSalhi MS, Siddiqui MK. Silver nanoparticles applications and human health. Clin Chim Acta. 2010;411:1841-1848.

46. Paquet P, Piérard GE. Interleukin-6 and the skin. Int Arch Allergy Immunol. 1996;109:308-317.
47. Park HS, Kim KH, Jang S, et al. Attenuation of allergic airway inflammation and hyperresponsivensess in a murine model of asthma by silver nanoparticles. Int J Nanomedicine. 2010;5:505-515.

48. Park HS, Kim KH, Jang S, et al. Silver nanoparticles modify VEGF signaling pathway and mucus hypersecretion in allergic airway inflammation. Int J Nanomedicine. 2010;7:1329-1343.

49. Sheikpranbabu S, Kalishwaralal K, Venkataraman D, Eom SH, Park J, Gurunathan S. Silver nanoparticles inhibit VEGF-and IL-1induced vascular permeability via Src dependent pathway in porcine retinal endothelial cells. J Nanobiotechnology. 2009; 7:8.

50. Lara HH, Ayala-Nunez NV, Ixtepan-Turrent L, RodriguezPadilla C. Model of antiviral action of silver nanoparticles against HIV-1. J Nanobiotechnology. 2010;8:1.

51. Lee YC, Lee HK. Vascular endothelial growth factor in patients with acute asthma. J Allergy Clin Immunol. 2001;107:1106.

52. Nolan A, Kobayashi H, Naveed B, et al. Differential role for CD80 and CD86 in the regulation of the innate immune response in murine polymicrobial sepsis. PLoS One. 2009;4:e6600.

53. Orabona C, Grohmann U, Belladonna ML, Fallarino F, Vacca C. CD28 induces immunostimulatory signals in dendritic cells via CD80 and CD86. Nat Immunol. 2004;5:1134-1142.

54. Sharpe AH, Freeman GJ. The B7-CD28 superfamily. Nat Rev Immunol. 2002;2:116-126.

55. Grohmann U, Orabona C, Fallarino F, et al. CTLA-4-Ig regulates tryptophan catabolism in vivo. Nat Immunol. 2002;3:1097-1101.

56. Nolan A, Weiden M, Kelly A, et al. CD40 and CD80/86 act synergistically to regulate inflammation and mortality in polymicrobial sepsis. Am J Respir Crit Care Med. 2008;177:301-308.
International Journal of Nanomedicine

\section{Publish your work in this journal}

The International Journal of Nanomedicine is an international, peerreviewed journal focusing on the application of nanotechnology in diagnostics, therapeutics, and drug delivery systems throughout the biomedical field. This journal is indexed on PubMed Central, MedLine, CAS, SciSearch $\AA$, Current Contents ${ }^{\circledR} /$ Clinical Medicine,

\section{Dovepress}

Journal Citation Reports/Science Edition, EMBase, Scopus and the Elsevier Bibliographic databases. The manuscript management system is completely online and includes a very quick and fair peer-review system, which is all easy to use. Visit http://www.dovepress.com/ testimonials.php to read real quotes from published authors. 